\title{
Perspectives
}

\section{Summer English Language and Cultural Studies Programs: Points to Ponder}

John Taplin and Lynn Wyton

In recent years there has been a steady increase across Canada in the number of short-term programs in English language and cultural studies. Typically, these programs are run in the summer months for groups of students from non-English-speaking countries for periods ranging from three to six weeks. Such programs provide visiting students with English classes, as well as the opportunity to learn about Canadian culture through field trips and accommodation in home stays or on-campus residences.

Despite the growth in the number and variety of these programs, there is little literature on the issues associated with the administration of such programs. Although we stress that each program must be examined in its own context, we wish to highlight a number of factors for consideration by administrators and instructors who are operating, or planning to operate, such programs.

\section{The Context}

We begin with an overview of the two programs with which we have experience at the University of Lethbridge in southern Alberta. The first is an "exchange" in the truest sense. As a component of the twinning agreement that was signed by the governments of Alberta and Hokkaido in 1980, our university and a private Japanese university host student groups of 15-20 for short-term visits. In alternating summers each university sends a group of students to the other for three weeks of language and cultural studies. The host institution also arranges home stays for the visiting students.

The second program has run each summer since 1991. A Japanese Christian girls' high school sends a group of 30-32 students aged between 15 and 17 years with two accompanying teachers for three weeks of English language study and experiences in Canadian culture. These students are also accommodated in local home stays, which are coordinated by a group of local churches.

\section{Motivation}

The participating institutions share the desire for students to have a practical and positive language learning experience. Classroom instruction is augmented by trips to local points of interest or further afield. Undoubtedly the 
greatest insight into Canadian life is offered by home stay families who allow students to participate in their lives on a daily basis. For the Japanese this is an integral component of short-term programs. For the Canadian hosts, as well as the visiting students, there is an opportunity to exchange cultural information. In fact the intercultural awareness that is furthered at the community level is one of the most positive by-products of such programs.

Several factors motivate institutions to become involved in short-term programs for groups. The laudable goal of promoting international understanding and goodwill has to be considered in the light of realistic budget expectations. These expectations are dependent on the nature of the relationship with overseas institutions and the role such programs play in the context of a language centre's total operation. Although the overall intention is to ensure a quality experience for all participants irrespective of the program, there are different aspects to an administrative arrangement that is characterized by a business contract rather than one that exists as part of an exchange agreement.

\section{Administration}

As in any business arrangements, the initial terms for setting up a short-term program are vital to the establishment of working relationships between institutions. The nature of both the agreement and the contract in the first instance create a precedent for continued dealings. Negotiations will become complicated if either participating party desires to make significant changes to the arrangement formulated for the first contract. Cultural dimensions will affect business negotiations from the start. In the Japanese style of business, more time is spent on consensus building than is usual in the West. As this can be a lengthy process, administrators of short-term programs must allow significant lead time for planning. Once the working relationship has been established, it is undesirable from the Japanese perspective for major changes to be implemented in subsequent years. Administration of such programs is most intensive in the summer just prior to and while the group is here.

Several other issues also need to be considered in the administration of a short-term program:

- Is an institution dealing directly with the institution sending the students? If another party such as a travel agency is involved, triangular rather than bilateral arrangements will take more time.

- Is there full disclosure of all the details of the arrangements between the key players? What level of budget specificity will be provided to the sending institution? A highly specific budget provided to the sending institution may lead to lengthy discussions about each line item.

- How flexible will an institution be in responding to requests? We have found it helpful to formulate a contract with the Japanese high school 
in which we outline specifically what our university is responsible for and what the sending institution manages. Thus there is a clear demarcation of responsibilities. Avoiding fatigue for all participants from both the host and sending institution is a primary concern in program planning.

It is also important to remember that there are particular dynamics at play when programs are run for students who are not part of an institution's regular enrollment. A helpful article that was published recently in Australia's ELICOS (English Language Intensive Courses for Overseas Students) Association Journal noted that, "Unlike students, paying clients are not fully absorbed within the institution and can therefore exercise rights which are not subject to systems of institutional control" (Crichton, 1994, p. 10). In short-term programs the client role is most visibly represented by the accompanying teachers, who have key responsibilities as chaperones. Generally, when they arrive, they wish to confirm the arrangements between participating parties. Thus it is important to address these concerns in the context of the contract with the sending institution. Coordination with home stays and keen attention to myriad details concerning the instructional and off-campus components of the program are vital. Because of the number of players in a short-term program and the involvement of people from the surrounding community, any mistakes tend to be highly visible. The implication is that attention to detail is crucial to a program's overall success.

\section{Staffing}

The focus in this section is on the level of staffing that the host institution requires. Our experience has been to host no more than 32 students and two teachers at any one time, although, in summer 1994 we hosted two groups in a consecutive six-week period. The challenges in overlapping two programs just on one day increased our work load significantly. When hosting a greater number of students and/or operating more than one short-term program simultaneously, institutions have greater logistical concerns.

We advise those responsible for a summer program to conduct an inventory of all the instructional and administrative needs. As well as an administrative coordinator, it has been extremely productive for us to hire a full-time undergraduate student for the projects. We have found that students who have intercultural experiences and interests, as well as career goals in ESL, are suitable for working with visiting students. The time spent in training a student can be amply rewarded, especially if a student can be hired over consecutive summers. Also helpful are SEED and STEP grants that the federal and provincial governments make available for student employment.

We have found it beneficial to keep administrative and instructional duties for staff distinct. As our instructors work with the visiting high school students an average of four hours a day in the classroom, they do not have a 
lot of time to be involved in the planning of the other activities. However, communication among all staff members is essential, and a brief troubleshooting session before each day's program is a definite asset. A staff that is clear on its roles and approaches activities with a spirit of collaboration and a sense of humor is as important to short-term programs as to programs in general.

\section{Curriculum}

Institutions that send their students overseas for short-term programs want learning experiences that will aid the maturation and English language improvement of their students. This has been borne out in our experiences working with two Japanese institutions. Japanese high school students usually have at least six years of exposure to English language instruction. Because of cultural patterns of learning and the fact that they learn in an EFL context, they tend to have a passive knowledge of English with an emphasis on formal grammar. Thus when they arrive in English-speaking Canada the Japanese students are beset by the full implications of English language immersion.

The realities of the short-term immersion experience drive curriculum choices. Largely as a result of the orientation they have received before leaving Japan, we find students are receptive to new experiences and ways of learning. Nevertheless, program planners should not overestimate the impact of classroom instruction. There is no question that what students learn in their home stays, in both a linguistic and a cultural sense, stays with them as their most compelling memory of their time in Canada. In our curriculum we have attempted to provide students with language tools and strategies to help them cope with living with English-speaking families. In addition, the cultural content of afternoon activities is reflected in the classroom lessons. We feel it is important to expose students to the multicultural realities of Canada. For example, when students experience aspects of local native culture on field trips, they are prepared through a basic orientation in the morning classes.

In essence, the curriculum has an experiential basis. As stated by Eyring (1991), "Providing real or quasi-real life experiences allows more opportunities for language learners to receive input. Interacting and cooperating with others allows opportunities for feedback on one's own language learning and humanizes the learning process" (p. 347). In smaller classes than they are used to in Japan, students practice functional and relevant English with each other. The instructors encourage the students to take more risks with the language so that they will feel more comfortable with their use of English outside the sheltered confines of the classroom. Students also respond enthusiastically to computer-assisted instruction, especially in tasks such as drafting letters in English to their friends through the use of word process- 
ing. Somewhat surprisingly, our students have had minimal experience of this in Japan.

Instructors must be aware that fatigue may impinge on the students' classroom performance. As anyone who has experienced a new culture knows, there is a certain level of exhaustion that accumulates from a combination of jet lag and novel stimuli crowding the senses. It is important to be realistic about what the students can accomplish in a short period.

\section{Safety}

One of our overriding concerns when planning the curriculum for our programs is safety. Understandably, host staff, parents, and Japanese chaperones are concerned for the security of the students on their overseas adventure. In planning both programs we attempt to strike a balance between interesting educational experiences and safety. The high school group demands particular care as the students are younger and the traditional Japanese emphasis on security is even more acute when women are present.

To alleviate concerns we attempt to exercise extra caution when planning the cultural studies program where some attractive but risky options are available for activities. Rather than a rafting trip, then, we have planned more sedate hikes by the side of a river or lake. Transportation for these events offers a potential problem, which we try to alleviate by using professional drivers whenever possible. They are asked to exercise extreme caution when driving in order not to frighten our guests.

Further challenges can emerge if volunteers are included in the program. They will need to be informed, for example, to take extra care when using their own vehicles and to adopt a more cautious stance than normal even in mundane activities. Although volunteers are usually involved in some of the more relaxed activities, they need to know that they also fulfill a supervisory role in the program. Thus our program aides receive a list of guidelines that helps them to remember some of the details for which they are responsible. After using these procedures, we can say with some confidence that no Japanese student ever traveled in a volunteer's car without a carefully fastened seat belt. Ultimately, such practices can assist in avoiding embarrassing or upsetting situations during a visit.

\section{Home Stays}

The organization of home stays adds significantly to the administration of the program. Program planners should address two main questions on this issue: Who is responsible for the home stay component? Will home stay families be paid to cover their costs, or will they be volunteers?

Both of the projects with which we are associated have a home stay component, but, as mentioned above, the responsibility for organizing the billets is completed by a local community group for the high school students. 
A comparison of the work load for the two programs clearly reveals that the time spent arranging the home stays for the university group is approximately equal to the time required to organize the rest of the program. Especially in the prearrival planning stages, accommodation arrangements require considerable time and energy dedicated to advertising, selecting, and informing people of our expectations. Maintaining contact with those billets and their guests once the program begins then requires regular communication with a varied group of people who justifiably demand a good deal of attention. Because so much of a program's success depends on a pleasant home stay environment, we consider the energy we put into this component of our programs to be a vital investment.

\section{Cultural Factors}

Any intercultural experience includes elements of uncertainty. On a leisure trip this may mean missing an exit on an unfamiliar roadway. The stakes are considerably higher, though, in an intercultural business venture where personal and professional reputations can affect interactions. The discussions are often further influenced by the anxiety that both sides can feel when attempting to arrange an agreement with few personal or cultural ties between parties. Ironically, the parties may inadvertently worsen tension by the very mechanisms that they use to ease the inevitable feelings of worry and doubt. To illustrate, it takes some patience on the part of a typical Westerner to sit quietly in mid-meeting and wait for a Japanese delegation to build consensus for a major decision. In the same way, Japanese visitors might find it difficult to understand why their Canadian counterparts cannot accommodate their requests for meetings that run into the late evening. Such examples do not depict cultural affronts; rather, they highlight some of the daily cross-cultural stressors that each party experiences.

Eventually, such seemingly insignificant events can strain both parties and compromise the vital goodwill in a program. To prevent this situation we have primarily used two strategies, the first of which relies on communication. To begin with we attempt to communicate with our visitors just where our program boundaries are set. In specific instances we may make exceptions to these parameters, but avoiding fatigue includes knowing when to politely refuse requests. We also try to develop purposeful communication among the host staff in order to proactively diffuse potentially difficult situations. By attempting to predict such possible trouble spots before they occur, and discussing those concerns with colleagues, some healthy crosscultural coping techniques emerge.

The second set of cross-cultural techniques is based on building stronger personal bonds between staff of the various institutions. Shortly after the arrival of the group, for example, we meet in a more social setting. This might include a dinner at a restaurant and a tour of some sites of interest in the host 
city. As the program develops we look for more opportunities to build understanding between visiting and hosting staff. In view of all the difficulties that can emerge in cross-cultural negotiations, networking of this variety can foster valuable patience and trust that can be critical in times of limited understanding.

Gifts for the students and accompanying teachers at the beginning, middle, and end of the program can also refresh visitors during a visit and take the edge off some of the stressors mentioned above. In our programs we attempt to offer practical gifts that coincide with an event in the curriculum. For example, University of Lethbridge ball caps were a significant addition to a day spent visiting a nearby lake and, judging from the pictures we received of the students on their return to Japan, were valued personal souvenirs. These personal touches can be built into the program budget, thus allowing them to be positive experiences rather than expensive program additions.

The result of these and other intercultural communication strategies is a healthy relationship between host and visiting staff.

\section{Conclusion}

Clearly, there is no blueprint for running short-term English language and cultural studies programs. While emphasizing that the particular dynamics at play with any specific group must be assessed, it is important to remember the plethora of factors to consider. In times of fiscal restraint administrators may look to short-term programs as a means of providing budget assistance to their overall operation. It is important, however, to look at all the dimensions that use up time and other resources in planning a quality program.

Hosting international guests is an honor for an institution and its surrounding community. It is also a considerable pleasure to work with young people during a particular period of exploration and wonder in their lives. We hope that by outlining some of these general points we can assist administrators and instructors to manage quality programs in their own contexts.

\section{Note}

An earlier version of this paper was presented at the ATESL 1994 Conference in Edmonton.

\section{The Authors}

John Taplin and Lynn Wyton both work at the English Language Centre, University of Lethbridge. John is the Coordinator of the Centre; Lynn is an instructor and Summer Programs Coordinator.

\section{References}

Crichton, J. (1994). Students as clients: Consequences for the construction of teaching roles. ELICOS Australia Journal, 12(2), 8-16. 
Eyring, J.L. (1991). Experiential language learning. In M. Celce-Murcia (Ed.), Teaching English as a second or foreign Language (pp. 346-359). Boston: Newbury House.

Runswick, S. (1993). The language learning styles and performance of Japanese speakers.

ELICOS Australia Journal, 11(1), 94-103.

Withey, J. (Ed. on behalf of the Canadian Chamber of Commerce in Japan). (1994). Doing business in Japan. Toronto: Key Porter.

Woollacott, M. (1995, January 29). A nation outside looking in. Guardian Weekly, 152(5), p. 7. 